\section{Understanding the early stages of calcium phosphate mineralization through hyperpolarized NMR techniques.}

\author{
TRISTAN GEORGES ${ }^{1}$, LUDOVICA EPASTO ${ }^{2}$, ALBINA \\ SELIMOVIĆ $^{2}$, DENNIS KURZBACH SR. ${ }^{2}$ AND THIERRY \\ AZAÏS SR. ${ }^{1}$
}

${ }^{1}$ Sorbonne Université

${ }^{2}$ Institute of Biological Chemistry

Presenting Author: tristan.georges@upmc.fr

Calcium phosphates are the main mineral component of vertebrate bones and teeth. Studying calcium phosphate nucleation can improve our understanding of physiological and pathological biomineralization events, and could therefore assist the design of superior biomaterials, e.g., in bone repair applications.

It has been suggested that calcium phosphate crystallization proceeds along non-classical crystallization pathways [1]. The latter assume that free ions in solution are in equilibrium with larger solute species dubbed prenucleation species (PNS). These clusters tend to aggregate with time, leading to the nucleation of an amorphous phase that later transforms into a crystalline phase, such as hydroxyapatite $\left(\mathrm{Ca}_{10}\left(\mathrm{PO}_{4}\right)_{6}(\mathrm{OH})_{2}\right)$ in case of bone mineralization [2]. However, little is known about PNS because of their nanometric size, their highly dynamic behavior and their short life-time [3].

In this work, we use both, liquid and solid-state NMR, in a time-resolved manner to highlight dynamics (PNS formation and aggregation rate, phosphate chemical exchange) and structural (size, chemical composition) details regarding PNS. We demonstrate that in SBF (simulated body fluid, a (meta)stable solution that mimics human blood plasma) free phosphates reach a full conversion into PNS after $>15$ hours, questioning the "stability" of SBF. ${ }^{31} \mathrm{P}$ diffusion ordered spectroscopy (DOSY) experiments and cryo-TEM observations revealed species of 2 $\mathrm{nm}$ in size that tends to aggregate into supramolecular structure of several hundreds of nm. Furthermore, we show through calcium ion and $\mathrm{pH}$ potentiometry that prenucleation clusters display a chemical composition close to calcium triphosphate units.

We also performed ${ }^{31} \mathrm{P}$ MAS DNP NMR on frozen solutions of calcium phosphate $(100 \mathrm{~K})$ to reveal structural details. We show that PNS bound phosphate can be distinguished from free phosphate on the basis of its chemical shift anisotropy (CSA) as it experiences a different coordination geometry in comparison to the bulk.

This work complements our previous real-time studies through dissolution-DNP on the formation of PNS under "fast" precipitation conditions [4].

References :

[1] De Yoreo et al. Science 2015, 349, 6247

[2] Dey et al. Nature materials 2010, 9(12), 1010-1014.

[3] Habraken, W. et al. Nature Communications 2012, 4, 1, 1-
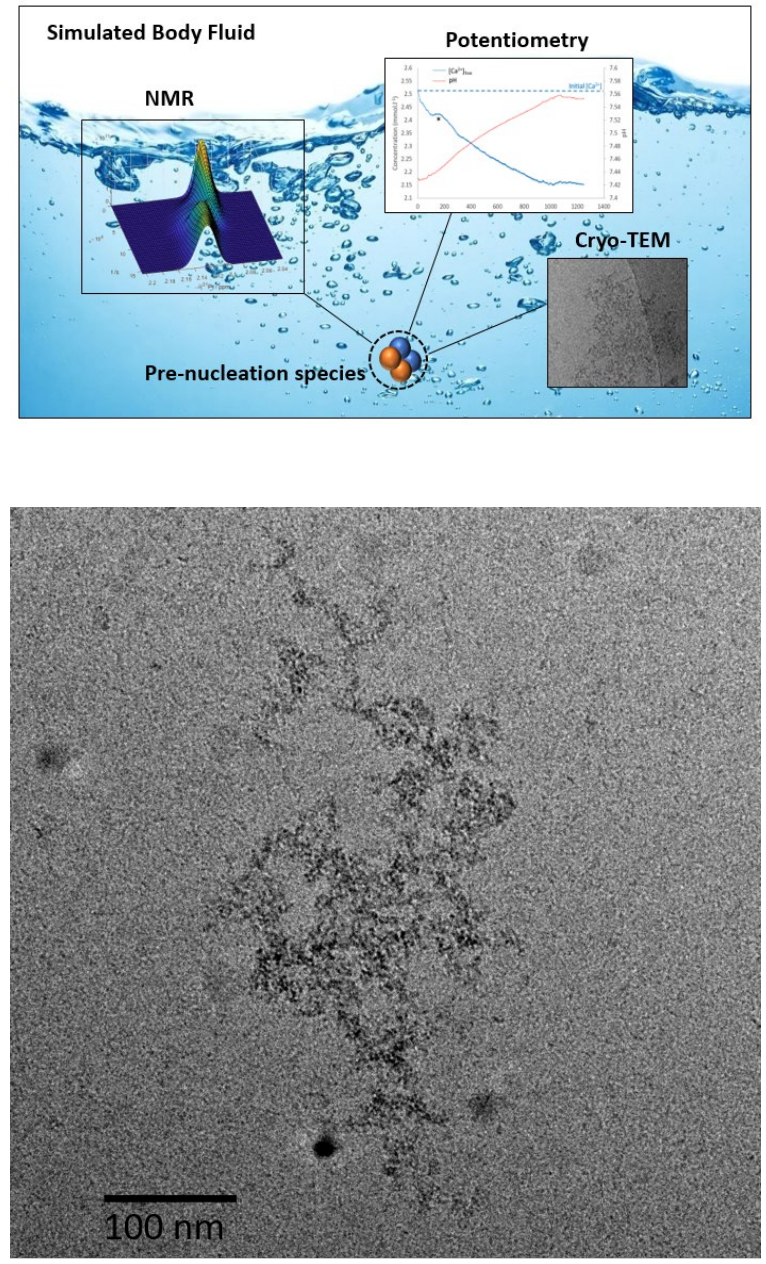